\title{
Preventing postoperative abdominal adhesions in a rat model with PEG-PCL-PEG hydrogel
}

This article was published in the following Dove Press journal:

International Journal of Nanomedicine

I February 2012

Number of times this article has been viewed

\author{
Bing Yang ${ }^{1,2 *}$ \\ ChangYang Gong ${ }^{*} *$ \\ Xia Zhao ${ }^{2}$ \\ ShengTao Zhou ${ }^{2}$ \\ ZhengYu $\mathrm{Li}^{2}$ \\ XiaoRong $\mathrm{Qi}^{2}$ \\ Qian Zhong ${ }^{2}$ \\ Feng Luo' \\ ZhiYong Qian'
}

'State Key Laboratory of Biotherapy, West China University Hospital, Sichuan University, Chengdu, People's Republic of China; ${ }^{2}$ Department of Gynecology and Obstetrics, West

China Second University Hospital, Sichuan University, Chengdu, People's Republic of China

*These authors contributed equally in this work
Correspondence: ZhiYong Qian State Key Laboratory of Biotherapy, West China Hospital, Sichuan University, Chengdu, 61004I, China

Tel +862885164063

Fax +862885164060

Email anderson-qian@।63.com
Background: Poly (ethylene glycol)-poly ( $\varepsilon$-caprolactone)-poly (ethylene glycol) (PEG-PCL-PEG, PECE) hydrogel has been demonstrated to be biocompatible and thermosensitive. In this study, its potential efficacy and mechanisms of preventing postsurgical abdominal adhesions were investigated.

Results: PECE hydrogel was transformed into gel state from sol state in less than 20 seconds at $37^{\circ} \mathrm{C}$. None of the animals treated with the hydrogel $(n=15)$ developed adhesions. In contrast, all untreated animals $(\mathrm{n}=15)$ had adhesions that could only be separated by sharp dissection $(P<0.001)$. The hydrogel adhered to the peritoneal wounds, gradually disappeared from the wounds within 7 days, and transformed into viscous fluid, being completely absorbed within 12 days. The parietal and visceral peritoneum were remesothelialized in about 5 and 9 days, respectively. The hydrogel prevented the formation of fibrinous adhesion and the invasion of fibroblasts. Also, along with the hydrogel degradation, a temporary inflammatory cell barrier was formed which could effectively delay the invasion of fibroblasts during the critical period of mesothelial regeneration.

Conclusion: The results suggested that PECE hydrogel could effectively prevent postsurgical intra-abdominal adhesions, which possibly result from the prevention of the fibrinous adhesion formation and the fibroblast invasion, the promotion of the remesothelialization, and the hydroflotation effect.

Keywords: anti-adhesion, thermosensitive, barrier, biocompatible

\section{Introduction}

Abdominal adhesions are a major cause of postsurgical morbidity and mortality and entail a substantial economic burden. Incidence ranges from $67 \%$ to $93 \%$ after general surgical abdominal procedures, and is even up to $97 \%$ after open gynecologic pelvic operations. ${ }^{1-3}$ Adhesions not only occur between the opposite peritoneal wounds, but also distant from the site of the preceding surgery. ${ }^{4}$ Although most intraperitoneal adhesions are usually asymptomatic, they can entail a broad range of secondary diseases such as infertility, chronic abdominal pain, bowel obstruction, and difficulties experienced during reoperative interventions. ${ }^{5-9}$

Peritoneal adhesions generally form in the early postoperative period. Therefore, unremitting efforts on this subject are focused on developing products used during laparotomy. Numerous drugs against fibrinous adhesion have been tested and have shown promise in animal models, but few have penetrated into clinical practice because of various side effects. ${ }^{10-12}$ One of the approaches to prevent adhesions is to apply polymer solution to separate the injured regions by the hydroflotation effect 
during peritoneal healing. ${ }^{11}$ However, for polymer solutions such as sodium hyaluronate (HA) and carboxymethylcellulose (CMC), the residence time at the site of administration is relatively brief. ${ }^{10}$ Another approach is to apply a synthetic solid membrane to keep tissue surfaces physically separated during the healing process. But for those membranes such as oxidized regenerated cellulose (Interceed $^{\circledR}$, Johnson \& Johnson Medical, Inc, New Brunswick, NJ), ${ }^{13}$ polytetrafluoroethylene, ${ }^{14}$ and HA-CMC (Seprafilm ${ }^{\circledR}$, Genzyme, Cambridge, MA), ${ }^{15}$ complete coverage of raw peritoneal surfaces can be difficult. In addition, application of those materials in laparoscopy can be cumbersome because of the difficulties in handling and fixation to the damaged tissue, which may also compromise their effectiveness as barrier systems. ${ }^{11,12}$ Furthermore, the risk of material residues for prolonged periods is one of the possible negative aspects of solid barriers. ${ }^{16}$

To conquer the abovementioned drawbacks, in-situ crosslinkable hydrogel systems (ie, polymer solutions that can form hydrogels when applied in situ) have been investigated. These crosslinkable hydrogel systems are prepared by chemical modification or cumbersome ultraviolet (UV) illumination. ${ }^{17-21}$ Recently, many crosslinkable hydrogels based on HA have been applied to the prevention of abdominal adhesions. However, the relatively long gelation time may be impractical. ${ }^{19}$ Additionally, some hydrogels which may retain locally for a comparatively long time could adversely affect the healing process of peritoneal wounds..$^{20,21}$ Hence, it is extremely necessary to find one kind of anti-adhesion agent which is not only effective and convenient to use, but also has a short gelation and retention time.

The authors of this paper have successfully prepared injectable poly (ethylene glycol)-poly ( $\varepsilon$-caprolactone)poly (ethylene glycol) (PEG-PCL-PEG, PECE) hydrogel containing basic components of PEG and PCL, which are biocompatible and have been used in several Food and Drug Administration-approved products. ${ }^{22}$ The prepared PECE hydrogels were proved to be thermosensitive, biocompatible, and bioabsorbable. ${ }^{22,23}$ In this present study, the aim was to assess its potential anti-adhesion efficacy and mechanisms in a rat model of surgical adhesion formation. Its potential toxicity was also investigated in vivo by histopathological observations.

\section{Materials and methods \\ Materials}

Materials used were: poly (ethylene glycol) methyl ether $\left(\mathrm{M}_{\mathrm{n}}=550\right)$ (Sigma-Aldrich, St Louis, MO), $\varepsilon$-caprolactone (Alfa Aesar, Ward Hill, MA), hexamethylene diisocyanate
(Sigma-Aldrich), stannous octoate (Sigma-Aldrich). All reagents were of analytic reagent grade and used as received.

\section{Preparation and characterization of PECE hydrogel}

Synthesis and characterization of PECE triblock copolymer

PECE copolymer was synthesized as described in previous work. ${ }^{22}$ Nuclear magnetic resonance ( $\left.{ }^{1} \mathrm{H}-\mathrm{NMR}\right)$ analysis (Varian 400 spectrometer, Varian Inc, Palo Alto, CA) and Fourier transform infrared (FTIR) spectroscopy (200SXV Infrared Spectrophotometer, Nicolet Co, Boston, MA) were used to characterize the prepared PECE copolymer.

\section{Preparation of PECE hydrogel}

The prepared PECE triblock copolymer was dissolved in normal saline (NS) at $60^{\circ} \mathrm{C}$ at a concentration of $25 \% \mathrm{w} / \mathrm{w}$ to form PECE hydrogel, and then PECE hydrogel was kept at $4^{\circ} \mathrm{C}$ until needed.

\section{Sol-gel-sol phase transition behavior}

The sol-gel-sol phase transition behavior of PECE hydrogel was investigated by rheometry (AR 2000EX, TA Instruments, New Castle, DE). The hydrogel was placed between parallel plates of $40 \mathrm{~mm}$ diameter and a gap of $31 \mu \mathrm{m}$. The data were collected under a controlled stress $\left(0.5 \mathrm{dyn} / \mathrm{cm}^{2}\right)$ and a frequency of $1.0 \mathrm{rad} / \mathrm{s}$. The heating rate was $2^{\circ} \mathrm{C} / \mathrm{min}$. Gelation times of the PECE hydrogel at $25^{\circ} \mathrm{C}$ and $37^{\circ} \mathrm{C}$ was also investigated by rheometry.

\section{The sidewall defect-cecum abrasion rat model and the application of PECE hydrogel}

All animal experiments were approved by the Institutional Animal Care and Use Committee of Sichuan University and were in compliance with all regulatory guidelines. Wistar albino female rats, weighing 240-260 g, were purchased from the Laboratory Animal Center of Sichuan University (Chengdu, China). All the animals were in quarantine for 1 week prior to treatment.

The sidewall defect-cecum abrasion rat model was established as described previously. ${ }^{24}$ Briefly, the rats were anesthetized by ketamine hydrochloride ( $60 \mathrm{mg} / \mathrm{kg}$ bodyweight) and were placed in the supine position, shaved in the abdominal area, prepared with iodine solution and ethanol, and then draped in a sterile fashion. An anterior midline incision was made through the abdominal wall and peritoneum. The cecum 
was identified, and the ventral side of the cecum was abraded with surgical gauze until an area of $3 \mathrm{~cm}^{2}$ was deserosalized as evidenced by punctuate bleeding without hemostasis. The damaged cecum was returned to the abdominal cavity, and then a $2 \mathrm{~cm} \times 3 \mathrm{~cm}$ apposing parietal peritoneal defect with punctate hemorrhage was created using a scalpel in the right lateral peritoneal surface of the abdominal wall. The two injured surfaces were approximated with $3 / 0$ silk suture to induce adhesions as the cecum in rats is too floppy.

The anti-adhesion efficacy of PECE hydrogel was evaluated 15 days after the operation. All rats were randomly divided into two groups and received $1 \mathrm{~mL}$ NS or

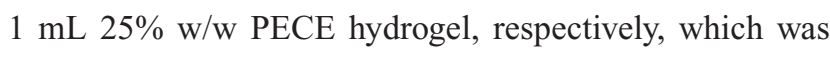
extruded through a 25 -gauge needle to uniformly paint both the raw surfaces. The incision was closed in two layers with $3 / 0$ surgical silk suture. On day 15 , the animals were sacrificed, and the adhesion formation was evaluated according to the widely used standard adhesion scoring system as follows: ${ }^{24-26}$ score 0 , no adhesion; score 1 , one thin filmy adhesion; score 2, definite localized adhesions; score 3, dense multiple visceral adhesions; score 4 , dense adhesions extending abdominal wall. The scoring was determined by two observers in a blinded manner.

Finally the potential anti-adhesion mechanism of PECE hydrogel was explored. The above procedures were repeated and the NS-treated rats were used as control to dynamically observe the adhesion formations at 1, 3, and 5 days after treatment. Meanwhile, the hydrogel degradation and the healing process of peritoneal wounds were dynamically observed through dissecting the hydrogel-treated rats at $1,3,5,7,9$, and 12 days after treatment.

\section{Tissue preparation}

Specimens were taken from the damaged cecum, damaged abdominal wall, and adhesion tissues contained in abdominal wall and cecum, and the specimen was carefully divided into two pieces. One was assigned for histopathological examination, and another for scanning electron microscopy (SEM) examination. For histopathological examination, the obtained tissues were fixed immediately in $4 \%$ paraformaldehyde in phosphate buffered saline (PBS) $(\mathrm{pH}=7.4)$ for 72 hours and embedded in paraffin. These tissues were then serially sectioned, stained with hematoxylin and eosin (H\&E) and Masson trichrome staining. All slides were analyzed using light microscopy (Olympus BX 45, Olympus, Hamburg, Germany) by two pathologists in a blinded manner. For SEM, the obtained tissues were fixed immediately with $2.5 \%$ glutaraldehyde in PBS and gradient dehydrated with ethanol.
The tissue samples were then dehydrated in a critical point apparatus, and were examined by scanning electron microscope (JSM-5900LV, JEOL, Japan) after a gold sputter coating.

\section{Toxicity assessment}

To evaluate possible side effects in PECE hydrogel-treated rats, all the animals were observed after administration of PECE hydrogel, including general condition (activity, energy, hair, feces, behavior pattern, and other clinical signs), bodyweight, and mortality. After sacrificing, various organs (heart, liver, spleen, lung, kidney, stomach, intestine, and brain) were harvested and fixed in $4 \%$ paraformaldehyde in PBS. These tissues were sectioned, stained with H\&E, and observed by two pathologists in a blinded manner.

\section{Statistical analysis}

Adhesion scores did not always follow a normal distribution. Statistical inferences were made using Mann-Whitney U-tests, or Fisher's exact test, using SPSS 10.0 (SPSS Inc, Chicago, IL) software. A $P$-value $<0.05$ on a two-tailed test was considered statistically significant.

\section{Results \\ Characterization of PECE hydrogel Characterization of PECE copolymer}

FTIR and ${ }^{1} \mathrm{H}-\mathrm{NMR}$ were used to characterize the chemical structure of the PECE copolymer. ${ }^{22,27}$ The $\mathrm{M}_{\mathrm{n}}$ and PEG/PCL block ratio of PECE triblock copolymer calculated from ${ }^{1} \mathrm{H}-\mathrm{NMR}$ spectra was 3630 and 1100/2530 respectively. FTIR and ${ }^{1} \mathrm{H}-\mathrm{NMR}$ results indicated that PECE triblock copolymer was prepared successfully.

\section{Temperature-dependent sol-gel-sol} transition behavior by rheology

The prepared PECE triblock copolymer was dissolved in NS at the designated temperature and at a concentration of $25 \% \mathrm{w} / \mathrm{w}$ to form PECE hydrogel, and then PECE hydrogel was sucked into a $1 \mathrm{~mL}$ syringe and kept at $4{ }^{\circ} \mathrm{C}$ until use (Figure 1A).

Gelation temperature was defined as the temperature at which storage modulus $\left(\mathrm{G}^{\prime}\right)$ and loss modulus $\left(\mathrm{G}^{\prime \prime}\right)$ were equal. Figure 1B shows the change in storage modulus $\left(\mathrm{G}^{\prime}\right)$ and loss modulus $\left(\mathrm{G}^{\prime \prime}\right)$ of PECE hydrogel $(25 \% \mathrm{w} / \mathrm{w})$ as a function of temperature. The $\mathrm{G}^{\prime}$ in sol state was less than $10 \mathrm{~Pa}$ and increased abruptly during the sol-gel transition at approximately $24^{\circ} \mathrm{C}$. When the temperature was $37^{\circ} \mathrm{C}, \mathrm{G}^{\prime}$ reached almost $600 \mathrm{~Pa}$. Then, the dramatic decrease of $\mathrm{G}^{\prime}$ 

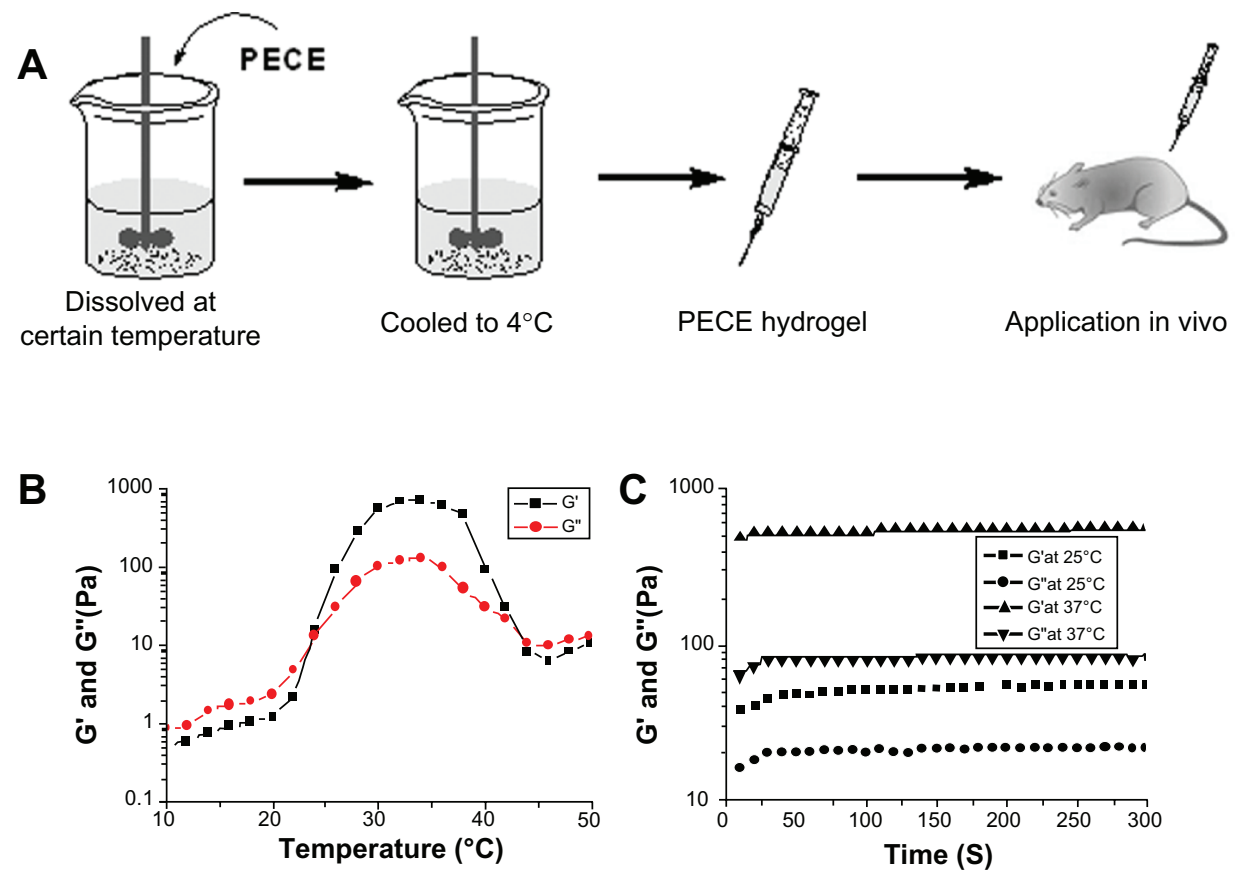

Figure I (A) Schematic illustration of PECE hydrogel for in vivo application. (B) Rheology analysis of PECE hydrogel as a function of temperature. (C) Time dependence of storage modulus $G^{\prime}$ and loss modulus $G^{\prime \prime}$ at different temperatures.

Abbreviation: PECE, poly (ethylene glycol)-poly ( $\varepsilon$-caprolactone)-poly (ethylene glycol) (PEG-PCL-PEG).

at about $42^{\circ} \mathrm{C}$ demonstrated the gel-sol transition of PECE hydrogel.

Gelation time was defined as the time when $\mathrm{G}^{\prime}$ became higher than $G^{\prime \prime}$ and reached a steady state. It reflected the changes in $\mathrm{G}^{\prime}$ and $\mathrm{G}^{\prime \prime}$ during the gelation process and stood for the gelation speed and gel intensity. Figure $1 \mathrm{~B}$ shows that the gelation temperature was $24^{\circ} \mathrm{C}$. In Figure $1 \mathrm{C}$, the gel was formed in less than 50 seconds at $25^{\circ} \mathrm{C}$, which is higher than the sol-gel transition temperature. When the temperature increased to $37^{\circ} \mathrm{C}$, gel formed in less than 20 seconds. During the gelation process, $G^{\prime}$ and $G^{\prime \prime}$ increased gradually and eventually reached a plateau, at which $G^{\prime}$ was significantly higher than $G^{\prime \prime}$. This indicated that the PECE hydrogel system displayed a predominantly solid-like behavior at body temperature.

\section{The anti-adhesion effects of PECE hydrogel in the rat model}

A total of 30 rats received laparotomies as described in the methods section (Figure 2A), were enrolled in this analysis, and randomly assigned into the NS or the PECE hydrogel group, respectively. A volume of $1 \mathrm{~mL}$ of $25 \% \mathrm{w} / \mathrm{w}$ PECE hydrogel was uniformly painted on both the wounds, and it quickly transformed into a gel state (Figure 2B).

The adhesion scores for all animals on the 15 th postoperative day are given in Table 1 . All rats $(100 \%)$ in the NS group developed score-4 adhesions (Figure 2C), indicating the adhesions of the cecum were directly apposed to the abdominal wall excision. Furthermore, intestine segment or omental adhesions to the sutured midline incisions were also observed in two and five cases, respectively. However, none of the 15 animals treated with the hydrogel developed adhesions (Figure 2D) $(P<0.001$, Fisher's exact test). The median adhesion scores were significantly lower than those in the control group ( $P<0.001$, Mann-Whitney U-test). At the time of dissection, all hydrogel-treated parietal and visceral defects returned to normal, and no hydrogel residue was observed in the abdominal cavities of all rats (Figure 2D).

\section{Autopsy observation}

The preliminary efficacy of anti-adhesion of the hydrogel in the rat model lead the authors to repeat the above procedures and conduct autopsies at different times in order to explore the potential mechanism of anti-adhesion.

On gross examination, all rats $(n=9)$ in the NS group developed adhesions between the apposed wounds (Table 2). On the first day after the operation, those adhesions were so fragile that they could be easily dissected bluntly (Figure 3A), and they became slightly firm on day 3 (Figure 3B). Upon examination on day 5 , they became so dense that their separation needed sharp dissection (Figure 3C). As shown in Table 2, a small amount of peritoneal exudate was observed on day 1 (Figure 3A), which significantly reduced 


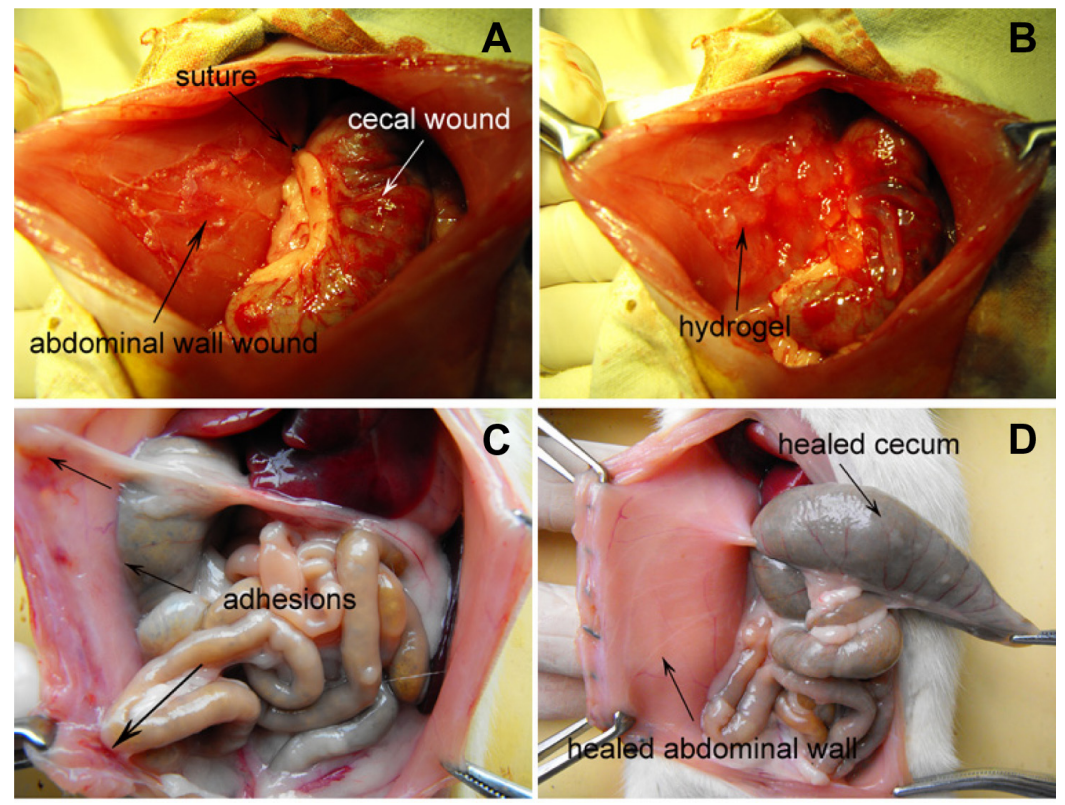

Figure 2 (A) The rat model of abdominal sidewall defect-cecum abrasion. (B) PECE hydrogel applied on the injured abdominal wall and cecum. (C) Score-4 adhesion was observed between the injured abdominal wall and cecum in an NS-treated rat; intestine segments and omentum adhesion to the sutured midline incisions were also observed. (D) No adhesion was observed in the PECE gel-treated group.

Abbreviations: NS, normal saline; PECE, poly (ethylene glycol)-poly ( $\varepsilon$-caprolactone)-poly (ethylene glycol) (PEG-PCL-PEG).

on day 3 (Figure 3B), and was almost absorbed on day 5 (Figure 3C).

In contrast to the NS group, none of hydrogel-treated rats $(n=18)$ developed adhesions (Table 2). On the first day after the treatment, the hydrogel, which was mixed with punctate hemorrhage, appeared red and adhered to the parietal and visceral wounds as discrete solid-like membranes, respectively (Figure 3D). A small amount of peritoneal exudate was also observed. The hydrogel gradually disappeared from the surfaces of the injured abdominal wall and the injured cecum within 3-5 days (Figure 3D-F) and 5-7 days (Figure 3D-G), respectively, transforming into viscous liquid. As shown in Table 2, the viscous liquid in the abdominal cavity was observed on day 3 (Figure 3E), gradually increased and

Table I Evaluation of peritoneal adhesions in the rat model

\begin{tabular}{|c|c|c|c|c|}
\hline \multirow[t]{2}{*}{ Adhesions } & \multicolumn{2}{|l|}{ NS $(n=15)$} & \multicolumn{2}{|c|}{$\operatorname{PECE}(n=15)$} \\
\hline & Frequency & Percentage & Frequency & Percentage \\
\hline Score 4 & 15 & 100 & 0 & 0 \\
\hline Score 3 & 0 & 0 & 0 & 0 \\
\hline Score 2 & 0 & 0 & 0 & 0 \\
\hline Score I & 0 & 0 & 0 & 0 \\
\hline Score 0 & 0 & 0 & 15 & 100 \\
\hline $\begin{array}{l}\text { Median } \\
\text { adhesion } \\
\text { score }\end{array}$ & $4(4-4)$ & & $0(0-0)$ & \\
\hline
\end{tabular}

Abbreviations: NS, normal saline; PECE, poly (ethylene glycol)-poly ( $\varepsilon$-caprolactone)-poly (ethylene glycol) (PEG-PCL-PEG). reached its peak on day 5 (Figure 3F), ending in complete absorption within postoperative day 12 (Figure 3G-I). With the hydrogel degradation and absorption, the injured parietal peritoneum and visceral peritoneum healed on days 5 and 9 (Figure 3F and H), respectively. The appearance of both surfaces had almost returned to normal 12 days after treatment (Figure 3I).

\section{Dynamic restoration of mesothelial cell layer of the peritoneal defects}

The peritoneum is composed of a monolayer of elongated, flattened, squamous-like mesothelial cells, with distinctive microvilli on the surfaces of the cells under SEM. ${ }^{28,29}$ SEM was used to observe the remesothelialization of peritoneal defects and the morphological changes of mesothelial cells. The surfaces of the stripped parietal peritoneum treated with PECE hydrogel at predetermined days are shown in Figures 4 and 5. Three days after treatment, the surface was still covered with a layer of hydrogel and was completely devoid of mesothelial cells (Figure 4A). The higher magnification image of SEM showed a covering of the PECE hydrogel on the meshwork of fibrin with small spherical inflammatory cells and erythrocytes, and without mesothelial cells (Figure 5A). Under SEM, it was striking that the injured surface was covered with a layer of elongated, flattened, squamous-like cells at day 5 (Figure 4B). The squamous-shaped cell junctions 
Table 2 Autopsy observation at different times in both groups

\begin{tabular}{|c|c|c|c|c|c|c|c|c|c|}
\hline \multirow[b]{2}{*}{ Days to dissection } & \multicolumn{3}{|l|}{ NS } & \multicolumn{6}{|c|}{ PECE } \\
\hline & 1 & 3 & 5 & I & 3 & 5 & 7 & 9 & 12 \\
\hline $\mathrm{n}$ & 3 & 3 & 3 & 3 & 3 & 3 & 3 & 3 & 3 \\
\hline Adhesion (n) & 3 & 3 & 3 & 0 & 0 & 0 & 0 & 0 & 0 \\
\hline Presence of residual gel on injured cecum & - & - & - & 3 & 3 & 3 & 0 & 0 & 0 \\
\hline Presence of residual gel on injured wall & - & - & - & 3 & 3 & 0 & 0 & 0 & 0 \\
\hline Mean volume of liquid $(\mathrm{mL})$ & 1.5 & 0.3 & 0 & 0.5 & 3.7 & 4.2 & 1.5 & 1.0 & 0 \\
\hline
\end{tabular}

Abbreviations: NS, normal saline; PECE, poly (ethylene glycol)-poly ( $\varepsilon$-caprolactone)-poly (ethylene glycol) (PEG-PCL-PEG).

on the surface became tight on day 7 (Figure 4C) and loose again on day 9 (Figure 4D). The morphology of these cells from day 5 to day 9 was noticeably different. These elongated, flattened, squamous-shaped cells had short microvilli on day 5 (Figure 5B). The number and length of microvilli increased on day 7 (Figure 5C), and many fine microvilli covered the surface of the cells on day 9 (Figure 5D).

Under SEM, the remesothelialization of the damaged visceral peritoneum treated with the hydrogel was similar to that of the parietal peritoneum, and needed about 9 days (data not shown).

\section{Transformation from fibrinous adhesion to fibrous adhesion in the NS group}

Usually, fibrinous adhesion requires three to five postoperative days to transform into fibrous adhesion. To investigate the anti-adhesion mechanism of PECE hydrogel, the rats treated with NS as control were sacrificed after the operation at indicated times, and the adhesion tissues involving the injured sidewall and cecum were stained with H\&E and Masson trichrome staining to clearly show the presence of collagen deposition and fibroblast activity. As shown in Figure 6, 1 day after the operation, the fibrinous adhesion was observed between the abdominal muscle wall and the cecal smooth muscle, with the infiltration of inflammatory cells (upper) and little collagen deposition (lower). After the operation, the increased infiltration with inflammatory cells (upper) and collagen deposition (lower) was observed between the abdominal muscle wall and the cecal smooth muscle.

\section{Fibrosis and remesothelialization of the peritoneal wounds treated with PECE hydrogel}

Rats treated with the hydrogel were sacrificed on indicated days, and the injured abdominal walls were stained with H\&E and Masson trichrome technique. Three days after
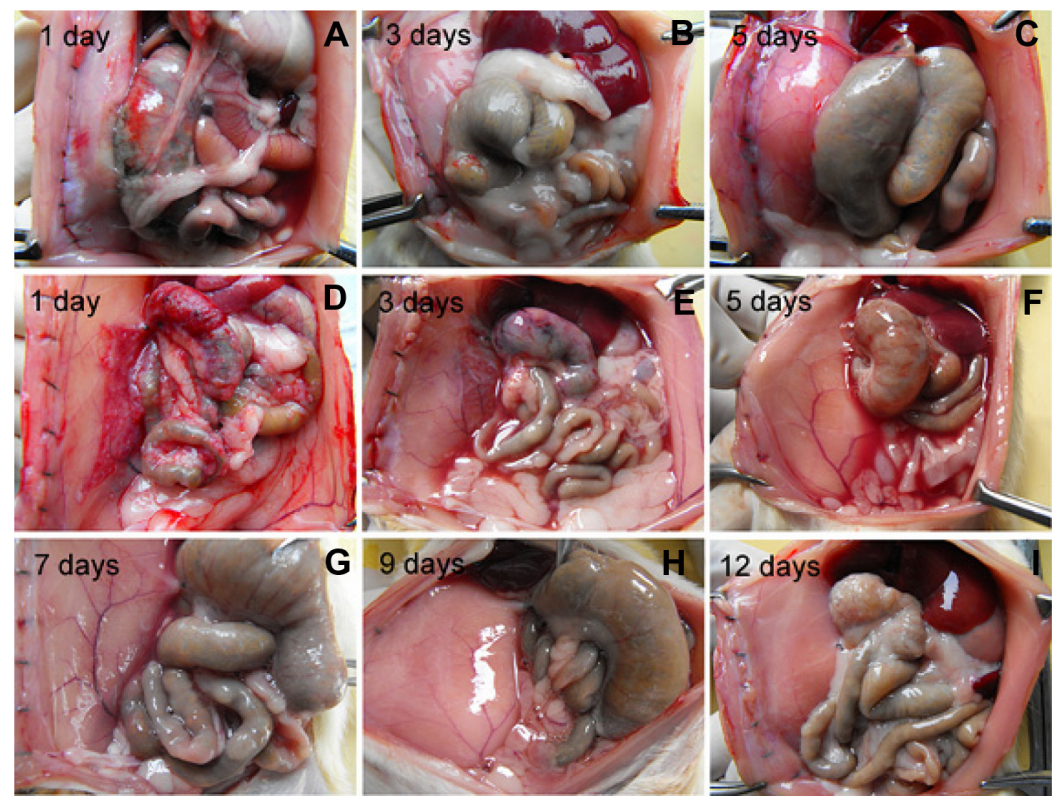

Figure 3 Autopsy observation in NS-treated group and hydrogel-treated group at different times. (A, B, and C) Adhesion formation on the indicated days in the NS group. (D, E, F, G, H, and I) The degradation of the adhered hydrogel and healing of the injured surfaces on the indicated days in the treatment group.

Abbreviation: NS, normal saline. 

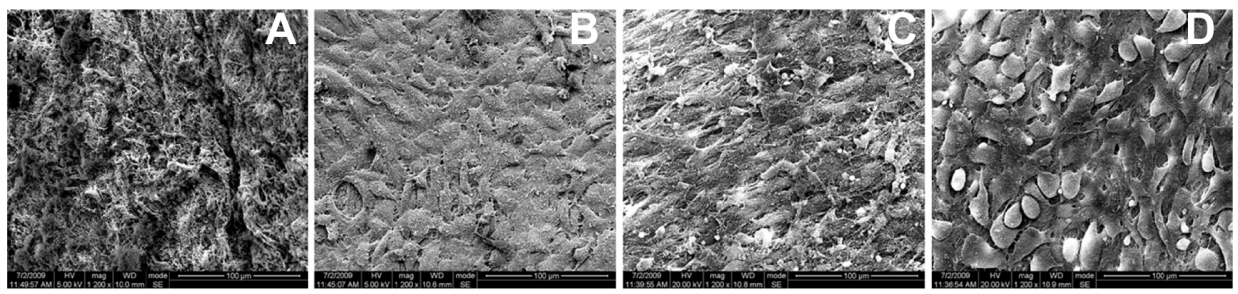

Figure 4 Scanning electron microscopy image of the surface of the stripped peritoneum. (A) The injured surface was covered by a layer of hydrogel on day 3. (B) The surface was covered with a layer of elongated, flattened, squamous-shaped cells on day 5 . The squamous-shaped cells junction became tight on day 7 (C) and loose again on day 9 (D).

treatment, the residual hydrogel, a pink layer of eosinophilic structure mixed mainly with macrophages and few foamy macrophages, was visible on the surface of the damaged abdominal wall with scattered collagen deposition beneath (Figure $7 \mathrm{~A}_{2}$ ). The foaminess of macrophages presumably was derived from their engulfing of polymeric debris (Figure $7 \mathrm{~A}_{1}$ ). Five days after treatment, with the disappearance of the hydrogel layer, the inflammatory cell layer, mainly composed of foamy macrophages and macrophages, appeared. There was a single layer of spindle-shaped mesothelial cells (Figure 7 $\mathrm{B}_{1}$ ) above the layer of inflammatory cells, while the collagen structure was restricted at the bottom of the layer of inflammatory cells (Figure $7 \mathrm{~B}_{2}$ ). The layer of inflammatory cells was gradually replaced by the collagen- and fibroblast-rich tissues over the next 7 days (Figure $7 \mathrm{C}_{1}-\mathrm{E}_{1}$ and $7 \mathrm{C}_{2}-\mathrm{E}_{2}$ ).

The pathological changes in damaged visceral peritoneum were similar to those in the parietal peritoneum (data not shown).

\section{Toxicity observation}

No gross abnormalities were observed in the hydrogeltreated rats. Histological examination of the substance of liver, spleen, kidney, heart, pancreas, lung, brain, intestine, and bone marrow did not reveal any significant differences between the treated and control groups. No apparent abnormalities were found in the mesothelial cell layers of other abdominal viscera in the hydrogel-treated animals.

\section{Discussion}

Intraperitoneal adhesion is considered to be an inevitable result of surgical trauma to the peritoneum. Trauma initiates an inflammatory response, followed by an increase in vascular permeability, release of fibrin-rich exudates and the formation of fibrinous adhesion. If fibrinolysis through the plasminogen-plasmin cascade is not effective enough, fibroblasts invade the fibrinous adhesion. Subsequently, collagen is deposited, leading to the formation of dense fibrous adhesions. ${ }^{10,30,31}$

Over the past few decades, numerous efforts have been taken against each step of pathogenesis of adhesion formation. However, those intensive studies failed to discover any pharmacological agents other than fibrinolytics or anti-inflammatory substances, which may still carry a high risk of bleeding and could potentially impair the healing process. ${ }^{11}$ Experts recommend using physical barrier systems to separate injured tissue surfaces during the first few postoperative days. ${ }^{11,15,32}$ The barrier systems, including viscous polymer solutions, preform solid sheets, and in-situ crosslinkable hydrogels, were designed to cover affected organs and reduce the contact between adjacent organs. For polymer solutions, the residence time at the site of administration is relatively short. ${ }^{10}$ For solid sheets, application of those materials can be complicated by difficulties in sheet handling and fixation to tissue and complete hemostasis, which may further compromise their efficacy as barrier systems. ${ }^{11,12}$ In addition, the material residues of solid barriers (over 31 days) might constitute an
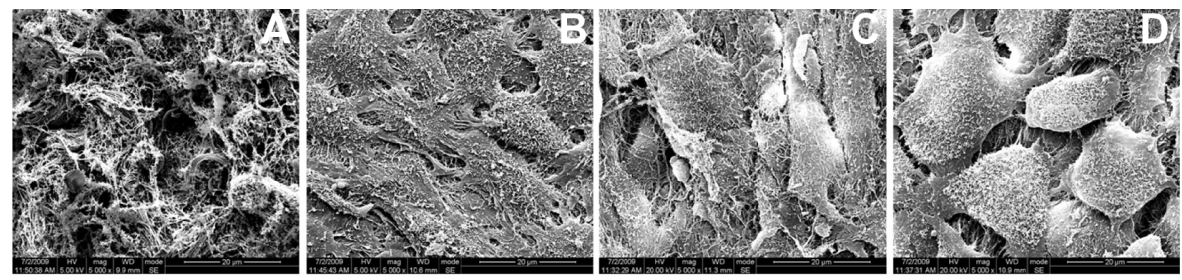

Figure 5 Scanning electron microscopy image of the surface of the stripped peritoneum (higher magnification of Figure 4). (A) The hydrogel covered the meshwork of fibrin, and several small spherical inflammatory cells were observed at day 3. (B) Large squamous-shaped cells over the surface and these cells had short microvilli. These microvilli increased and elongated at days 7 (C) and 9 (D). 


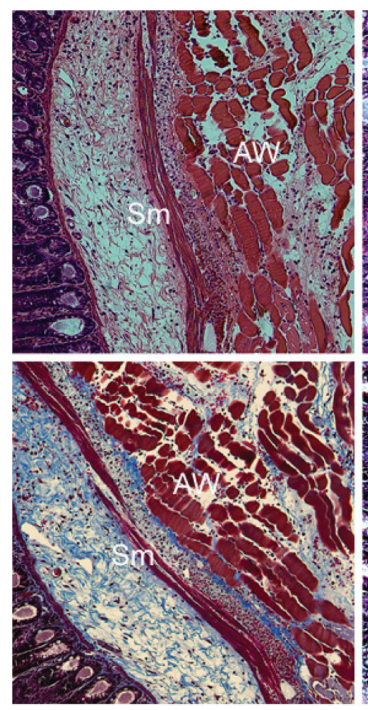

1

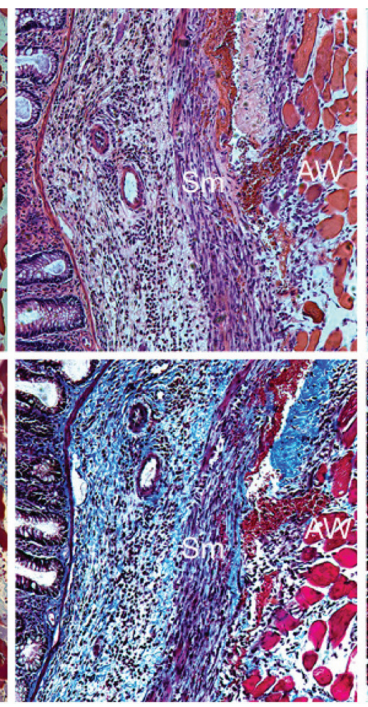

3

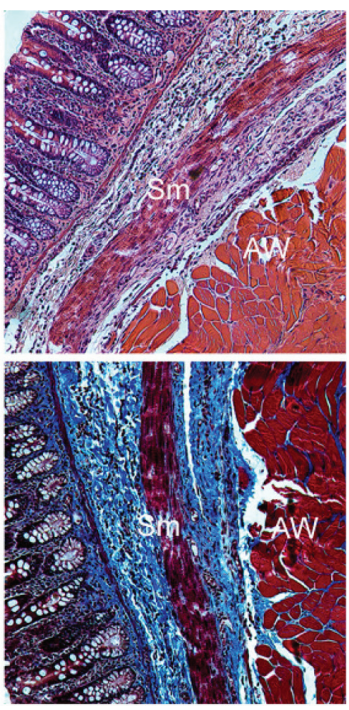

5

Time (d) after operation

Figure 6 Postsurgical adhesion formation at different times in rats treated with NS. Note that, along with days after the operation, infiltration with inflammatory cells (upper) and collagen deposition (lower) in both abdomen walls and cecal walls increased.

Abbreviations: AW, abdominal wall muscle; NS, normal saline; Sm, smooth muscle.

underlying threat to general wellbeing in humans. ${ }^{16}$ Recently, many in-situ crosslinkable hydrogels based on HA have been applied to the prevention of adhesions. ${ }^{17-21}$ However, their relatively long gelation time (5-45 minutes) or requirement of UV illumination may be impractical. ${ }^{17,19}$ Moreover, the material residues of these in-situ crosslinkable hydrogels, which can last for a relatively long time (over 7 days) at the application sites, could negatively affect the remesothelialization of peritoneal defects. ${ }^{20,21}$ Thus, the application of the present barrier systems was limited due to the abovementioned shortcomings.
The ideal barrier should not only be anti-adhesive, biocompatible, resorbable, and applicable through the laparoscope, but also should adhere to the traumatized surfaces without sutures or staples, even oozing surfaces. In addition, it should not interfere with remesothelialization. ${ }^{11,12}$ In previous work, the PECE hydrogel was proven to be injectable, biocompatible, and bioabsorbable. ${ }^{22,23}$ Results showed that the hydrogel could not only be adhered to the peritoneal wounds even with punctate hemorrhage but also effectively prevent postsurgical intra-abdominal adhesions. The key for preventing adhesions is the reconstruction of the neo-mesothelial cell layer.

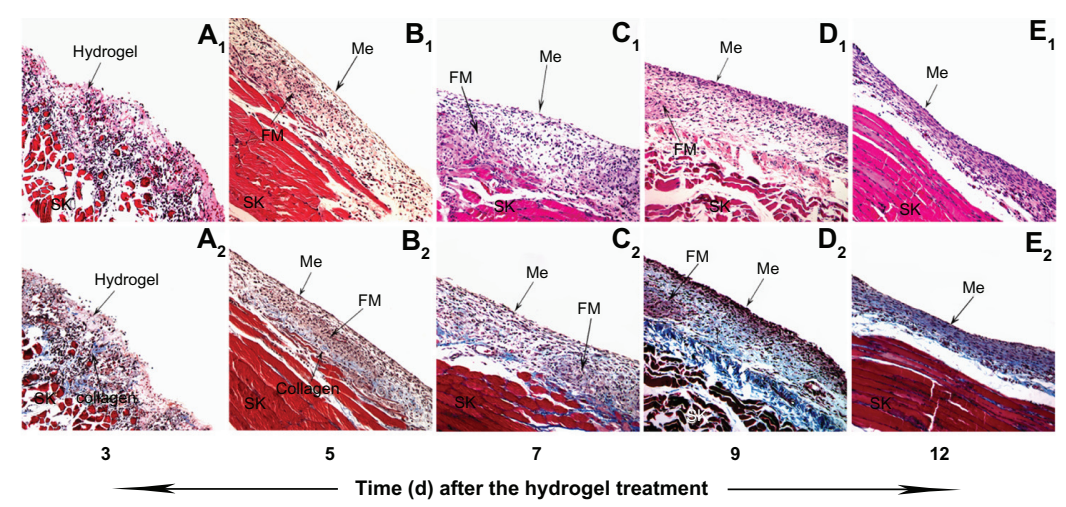

Figure 7 The fibrosis and the remesothelialization of the peritoneal wounds treated with PECE hydrogel. Three days after the treatment, a coating of the hydrogel on the injured surface of the abdominal wall, with infiltration of inflammatory cells $\left(\mathbf{A}_{1}, 200 \times\right)$ and scattered collagen beneath $\left(\mathbf{A}_{2}\right.$, 200x). Five days after the treatment, the layer of inflammatory cells composed mainly of foamy macrophages appeared, above which was a layer of spindle-shaped mesothelial cells (B $\mathbf{B}_{1}$, 200x), and under which was a collagen layer $\left(\mathbf{B}_{2}, 200 \times\right)$. Along with days, collagen- and fibroblast-rich tissues gradually increased and replaced the structure of the inflammatory cell layer $\left(\mathbf{C}_{1}\right.$, $\mathbf{C}_{2}$, $\mathbf{D}_{1}$, $\left.D_{2}, E_{1}, E_{2}, 200 \times\right)$.

Abbreviations: Me, mesothelial cell layer; SK, skeletal muscle; FM, foamy macrophages; PECE, poly (ethylene glycol)-poly ( $\varepsilon$-caprolactone)-poly (ethylene glycol) (PEG-PCL-PEG). 
In general, the remesothelialization requires about 5-6 days for the parietal peritoneum and about 5-8 days for the visceral mesothelium. ${ }^{11}$ The results of this present study showed that the PECE hydrogel-treated parietal and visceral peritoneum were remesothelialized within about 5 and 9 days, respectively, which indicated that the application of hydrogel did not noticeably impede the remesothelialization of parietal peritoneum and visceral mesothelium (Figures 4 and 5).

Avoiding fibrinous adhesion formation, preventing invasion of fibroblasts, and promoting remesothelialization play critical roles in adhesion prophylaxis. The results of this study showed that PECE hydrogel possesses some promising properties against the pathogenesis of adhesion formation, although this effect still remains metabolically elusive. It was supposedly achieved by the following mechanisms. At body temperature, the hydrogel adhered to the peritoneal wounds and separated the two damaged surfaces, thus preventing the formation of fibrinous adhesions between the adjacent peritoneal wounds and the invasion of fibroblasts. Subsequently, along with the gradual hydrogel degradation from the wounds, the recruited inflammatory cells, mainly composed of foamy macrophages and macrophages to the hydrogel-treated surfaces, formed a "second barrier" that could effectively delay the invasion of fibroblasts during the critical period of mesothelial regeneration. Mesothelial regeneration requires recruitment of macrophages to the wound surfaces, which will release mitogenic cytokines to activate and stimulate mesothelial cell proliferation. ${ }^{33-35}$ Although it is not known exactly where the neo-mesothelial cells come from, a number of apparent evidences have shown that the regenerating mesothelium does not rely on sub-mesothelial mesenchymal precursors. ${ }^{36}$ Instead, it is associated with the attachment of free-floating mesothelial cells to the injured peritoneal surfaces. ${ }^{37}$ Therefore, the results of this present study suggest that the second barrier mentioned above does not only delay the invasion of fibroblasts but also creates favorable conditions for the attachment, activation, and proliferation of the floating mesothelial cells during this critical period. After the remesothelialization, this second barrier gradually disappeared and was replaced by the collagen- and fibroblast-rich tissues. The neo-mesothelial cell layer was finally located on the basement built by fibrosis tissue (Figure 7).

In addition, there was an accumulation of viscous liquid in the abdominal cavity along with the hydrogel disappearance from the wounds, but the liquid was completely absorbed within 12 days after the operation. Even though its formation mechanism and components are far from clear, the impaired peritoneums completed their remesothelialization, and mesothelial cell morphology was restored, accompanied by the generation and absorption of viscous liquid. The results also suggested that the hydrogel has excellent biocompatibility. Also noted was that not only the treated injured surfaces but also the untreated sutured incisions did not suffer from any adhesion. On the contrary, in the control group, the adhesions were detected not only between both the opposite peritoneal wound surfaces but also between the sutured incisions and the normal peritoneum. Therefore, in addition to the abovementioned anti-adhesion mechanism, the viscous liquid arising from the hydrogel degradation might help avoid the direct contact between the opposite peritoneal wound surfaces as well as between normal peritoneum and the injured peritoneum by the hydroflotation effect.

The results of this study revealed that PECE hydrogel has a number of unique physicochemical properties that are desirable for preventing postoperative adhesions compared with existing barrier systems. First, it can be easily extruded through needles and adhere to affected sites without spatial restriction, and quickly form a pliable and durable membrane at body temperature, without requiring additional initiators or UV illumination. This feature also makes the hydrogel suitable for laparoscopic applications. Secondly, those inflammatory cells mainly composed of foamy macrophages and macrophages built up the second physical barrier due to the application of PECE-hydrogel. It helped to delay the invasion of fibroblasts and promote the remesothelialization. Another attractive characteristic is that the viscous fluid produced by the hydrogel degradation or dissolution might avoid the emergence of unexpected adhesions by the hydroflotation effect. Furthermore, the hydrogel can be cleared from the abdominal cavity in a relatively short time after the remesothelialization. Finally, although the damaged peritoneal defects with punctate hemorrhage were left in the models, it did not hamper the capability of the hydrogel to prevent adhesions.

In conclusion, PECE hydrogel presented in this work was suitable for application in the peritoneal cavity. Its great biocompatibility and biodegradability was proven in the rat model. Its physicochemical characteristics and distinctive degradation kinetics render it as a promising anti-adhesion candidate. Its powerful anti-adhesion effects were primarily achieved by preventing the formation of the fibrinous adhesion and the fibroblasts invasion, promoting the remesothelialization and the hydroflotation effect.

\section{Authors' contributions}

ZYQ, XZ, FL, BY and CYG designed the experiments. The research funding was supported by ZYQ and XZ. BY, CYG 
and XRQ carried out the experiments, analyzed the data, and wrote the manuscript; ZYQ, XZ, ZYL, STZ and QZ corrected the manuscript. XRQ and STZ participated in the animal test of the hydrogels. All authors approved and read the final manuscript.

\section{Acknowledgments}

This investigation was financially supported by National 863 Project (2007AA021804 and 2007AA021902), National Natural Science Foundation (NSFC20704027 and NSFC81071864), Specialized Research Fund for the Doctoral Program of Higher Education (200806100065), Sichuan Key Technology R\&D Program (2011SZ0219), and Chinese Key Basic Research Program (2010CB529906).

\section{Disclosure}

The authors declare that they have no competing interests.

\section{References}

1. Weibel MA, Majno G. Peritoneal adhesions and their relation to abdominal surgery. A postmortem study. Am J Surg. 1973;126:345-353.

2. Menzies D, Ellis H. Intestinal obstruction from adhesions - how big is the problem? Ann R Coll Surg Engl. 1990;72:60-63.

3. Group OLS. Postoperative adhesion development after operative laparoscopy: evaluation at early second-look procedures. Operative Laparoscopy Study Group. Fertil Steril. 1991;55:700-704.

4. Best CL, Rittenhouse D, Vasquez C, Norng T, Subias E, Sueldo CE. Evaluation of interceed(TC7) for reduction of postoperative adhesions in rabbits. Fertil Steril. 1992;58:817-820.

5. DeCherney AH, diZerega GS. Clinical problem of intraperitoneal postsurgical adhesion formation following general surgery and the use of adhesion prevention barriers. Surg Clin North Am. 1997;77:671-688.

6. Ray NF, Denton WG, Thamer M, Henderson SC, Perry S. Abdominal adhesiolysis: inpatient care and expenditures in the United States in 1994. J Am Coll Surg. 1998;186:1-9.

7. Coleman MG, McLain AD, Moran BJ. Impact of previous surgery on time taken for incision and division of adhesions during laparotomy. Dis Colon Rectum. 2000;43:1297-1299.

8. Parker MC, Ellis H, Moran BJ, et al. Postoperative adhesions: ten-year follow-up of 12,584 patients undergoing lower abdominal surgery. Dis Colon Rectum. 2001;44:822-829; discussion 829-830.

9. Fazio VW, Cohen Z, Fleshman JW, et al. Reduction in adhesive small-bowel obstruction by Seprafilm adhesion barrier after intestinal resection. Dis Colon Rectum. 2006;49:1-11.

10. Diamond MP. Reduction of de novo postsurgical adhesions by intraoperative precoating with Sepracoat (HAL-C) solution: a prospective, randomized, blinded, placebo-controlled multicenter study. The Sepracoat Adhesion Study Group. Fertil Steril. 1998;69:1067-1074.

11. Liakakos T, Thomakos N, Fine PM, Dervenis C, Young RL. Peritoneal adhesions: etiology, pathophysiology, and clinical significance. Recent advances in prevention and management. Dig Surg. 2001;18: 260-273.

12. Attard JA, MacLeanAR. Adhesive small bowel obstruction: epidemiology, biology and prevention. Can J Surg. 2007;50:291-300.

13. Prevention of postsurgical adhesions by INTERCEED(TC7), an absorbable adhesion barrier: a prospective randomized multicenter clinical study. INTERCEED(TC7) Adhesion Barrier Study Group. Fertil Steril. 1989;51:933-938.
14. Haney AF, Doty E. Murine peritoneal injury and de novo adhesion formation caused by oxidized-regenerated cellulose (Interceed [TC7]) but not expanded polytetrafluoroethylene (Gore-Tex Surgical Membrane). Fertil Steril. 1992;57:202-208.

15. Becker JM, Dayton MT, Fazio VW, et al. Prevention of postoperative abdominal adhesions by a sodium hyaluronate-based bioresorbable membrane: a prospective, randomized, double-blind multicenter study. J Am Coll Surg. 1996;183:297-306.

16. Wallwiener M, Brucker S, Hierlemann H, Brochhausen C, Solomayer E, Wallwiener C. Innovative barriers for peritoneal adhesion prevention: liquid or solid? A rat uterine horn model. Fertil Steril. 2006;86: 1266-1276.

17. Sawhney AS, Pathak CP, van Rensburg JJ, Dunn RC, Hubbell JA. Optimization of photopolymerized bioerodible hydrogel properties for adhesion prevention. J Biomed Mater Res. 1994;28:831-838.

18. Osada H, Takahashi K, Fujii TK, Tsunoda I, Satoh K. The effect of cross-linked hyaluronate hydrogel on the reduction of post-surgical adhesion reformation in rabbits. J Int Med Res. 1999;27:233-241.

19. Liu Y, Li H, Shu XZ, Gray SD, Prestwich GD. Crosslinked hyaluronan hydrogels containing mitomycin $\mathrm{C}$ reduce postoperative abdominal adhesions. Fertil Steril. 2005;83 Suppl 1:1275-1283.

20. Yeo Y, Highley CB, Bellas E, et al. In situ cross-linkable hyaluronic acid hydrogels prevent post-operative abdominal adhesions in a rabbit model. Biomaterials. 2006;27:4698-4705.

21. Yeo Y, Ito T, Bellas E, Christopher B, Robert M, Daniel SK. In situ cross-linkable hyaluronan hydrogels containing polymeric nanoparticles for preventing postsurgical adhesions. Ann Surg. 2007;245: 819-824.

22. Gong C, Shi S, Dong P, et al. Synthesis and characterization of PEG-PCL-PEG thermosensitive hydrogel. Int J Pharm. 2009;365: 89-99.

23. Gong CY, Wu QJ, Dong PW, et al. Acute toxicity evaluation of biodegradable in situ gel-forming controlled drug delivery system based on thermosensitive PEG-PCL-PEG hydrogel. J Biomed Mater Res B Appl Biomater. 2009;91:26-36.

24. Ersoy E, Ozturk V, Yazgan A, Ozdogan M, Gundogdu H. Effect of polylactic acid film barrier on intra-abdominal adhesion formation. J Surg Res. 2008;147:148-152.

25. Knightly JJ, Agostino D, Cliffton EE. The effect of fibrinolysin and heparin on the formation of peritoneal adhesions. Surgery. 1962;52:250-258.

26. Ersoy E, Ozturk V, Yazgan A, Ozdogan1 M, Gundogdu H. Comparison of the two types of bioresorbable barriers to prevent intra-abdominal adhesions in rats. J Gastrointest Surg. 2009;13:282-286.

27. Gong CY, Dong PW, Shi S, et al. Thermosensitive PEG-PCL-PEG hydrogel controlled drug delivery system: sol-gel-sol transition and in vitro drug release study. J Pharm Sci. 2009;98:3707-3717.

28. Mutsaers SE. Mesothelial cells: their structure, function and role in serosal repair. Respirology. 2002;7:171-191.

29. Nishioka Y, Miyazaki M, Abe K, et al. Regeneration of peritoneal mesothelium in a rat model of peritoneal fibrosis. Ren Fail. 2008;30: 97-105.

30. De Iaco PA, Stefanetti M, Pressato D, et al. A novel hyaluronan-based gel in laparoscopic adhesion prevention: preclinical evaluation in an animal model. Fertil Steril. 1998;69:318-323.

31. Ustun C, Yanik FF, Kocak I, et al. Effects of Ringer's lactate, medroxyprogesterone acetate, gonadotropin-releasing hormone analogue and its diluent on the prevention of postsurgical adhesion formation in rat models. Gynecol Obstet Invest. 1998;46:202-205.

32. Marana R, Catalano GF, Caruana P, Margutti F, Muzii L, Mancuso S. Postoperative adhesion formation and reproductive outcome using Interceed after ovarian surgery: a randomized trial in the rabbit model. Hum Reprod. 1997;12:1935-1938.

33. Fotev Z, Whitaker D, Papadimitriou JM. Role of macrophages in mesothelial healing. J Pathol. 1987;151:209-219. 
34. Mutsaers SE, McAnulty RJ, Laurent GJ, Versnel MA, Whitaker D, Papadimitriou JM. Cytokine regulation of mesothelial cell proliferation in vitro and in vivo. Eur J Cell Biol. 1997;72:24-29.

35. Mutsaers SE, Whitaker D, Papadimitriou JM. Stimulation of mesothelial cell proliferation by exudate macrophages enhances serosal wound healing in a murine model. Am J Pathol. 2002;160:681-692.
36. Mutsaers SE, Whitaker D, Papadimitriou JM. Mesothelial regeneration is not dependent on subserosal cells. J Pathol. 2000;190:86-92.

37. Foley-Comer AJ, Herrick SE, Al-Mishlab T, Prêle CM, Laurent GJ, Mutsaers SE. Evidence for incorporation of free-floating mesothelial cells as a mechanism of serosal healing. J Cell Sci. 2002;115: 1383-1389.

\section{Publish your work in this journal}

The International Journal of Nanomedicine is an international, peerreviewed journal focusing on the application of nanotechnology in diagnostics, therapeutics, and drug delivery systems throughout the biomedical field. This journal is indexed on PubMed Central, MedLine, CAS, SciSearch $®$, Current Contents $® /$ Clinical Medicine,
Journal Citation Reports/Science Edition, EMBase, Scopus and the Elsevier Bibliographic databases. The manuscript management system is completely online and includes a very quick and fair peer-review system, which is all easy to use. Visit http://www.dovepress.com/ testimonials.php to read real quotes from published authors.

Submit your manuscript here: http://www.dovepress.com/international-journal-of-nanomedicine-journal 